\title{
PREPRINT
}

\section{Physical activity cancels the negative impact of adverse childhood experiences and depression on functional dependence}

\author{
Matthieu P. Boisgontier ${ }^{1, *}$; Dan Orsholits ${ }^{2,3}$; Martina von $\operatorname{Arx}^{2,3}$; Stefan Sieber $^{2,3}$; Delphine S. Courvoisier ${ }^{4}$; Maura D. Iversen ${ }^{5-7}$; Stéphane \\ Cullati $^{2,3,4, \uparrow}$; Boris Cheval ${ }^{2,3,5, \uparrow}$ \\ ${ }^{1}$ University of British Columbia, Department of Physical Therapy, Canada $;{ }^{2}$ Centre for the Interdisciplinary Study of Gerontology and Vulnerability, \\ University of Geneva, Switzerland; 'Swiss NCCR "LIVES - Overcoming Vulnerability: Life Course Perspectives", University of Geneva, \\ Switzerland; ${ }^{4}$ Department of General Internal Medicine, Rehabilitation and Geriatrics, University of Geneva, Switzerland; ${ }^{5}$ Department of \\ Medicine, Section of Clinical Sciences, Brigham and Women's Hospital, Harvard Medical School, Boston, Massachusetts, United States of \\ America; ${ }^{6}$ Department of Physical Therapy, Movement and Rehabilitation Sciences, Northeastern University, Boston, Massachusetts, United States \\ of America ; ${ }^{7}$ Department of Women's and Children's Health, Karolinska Institute, Stockholm, Sweden; ${ }^{\dagger}$ Equal contribution; ${ }^{*}$ Corresponding author: \\ BoisgontierMatthieu@gmail.com; @MattBoisgontier (M.P. Boisgontier)
}

Citation: Boisgontier, M. P., Orsholits, D., von Arx, M., Sieber, S., Courvoisier, D., Iversen, M., Cullati, S., Cheval, B. (2018). Physical activity cancels the negative impact of adverse childhood experiences and depression on functional dependence. https://doi.org/10.31236/osf.io/ms2u7

\begin{abstract}
Adverse childhood experiences, depression, and functional dependence are inter-related. However, mechanisms underlying this relation remain unclear. Here, we investigated the potential of depression to mediate the effect of adverse childhood experiences on functional dependence in older age and whether physical activity moderated this mediation. Data from 25,775 adults aged $62 \pm 9$ years from the Survey of Health Ageing and Retirement in Europe (SHARE) was used in adjusted linear mixed-effect models to test whether depression mediated the associations between adverse childhood experiences and functional dependence in activities of daily living (ADL) and instrumental activities of daily living (IADL) and whether physical activity moderated these mediations. As expected, adverse childhood experiences were positively associated with ADL $(b=.040$ for 0 vs. 1 and $b=.067$ for 0 vs. $\geq 2$ adverse childhood experiences) and IADL ( $b=.046$ for 0 vs. 1 and $b=.076$ for 0 vs. $\geq 2$ adverse childhood experiences). Both associations were mediated by depression. Physical activity reduced the effect of adverse childhood experiences on depression ( $b=-.060$ for 0 vs. 1 and $b=-.135$ for 0 vs. $\geq 2$ adverse childhood experiences) and canceled the effect of depression on functional dependence in ADL $(b=.073)$ and IADL $(b=.100)$, thereby eliminating the effect of adverse childhood experiences on functional dependence. Physical activity cancels the impact of adverse childhood experiences on functional dependence. In inactive individuals, the effect of adverse childhood experiences on functional dependence (ADL and IADL) is mediated by depression.
\end{abstract}

\section{INTRODUCTION}

In a recent systematic review, Hughes et al. (2017) demonstrated that adverse childhood experiences, such as neglect and household dysfunction, were associated with the twenty-three health outcomes identified in their review (e.g., depression, anxiety, physical inactivity, obesity). While these results highlight the pervasive harms that adverse childhood experiences place on multiple dimensions of health, they do not reveal the nature of the relations linking adverse childhood experiences and health outcomes. Assessing whether these relations are direct or indirect (i.e., explained by an intermediary variable) and whether they can be attenuated or eliminated through other factors would refine our understanding of the mechanisms underlying these relations.

Functional dependence is one of the health outcomes that has been associated with adverse childhood experiences (Laditka and Laditka, 2018; Amemiya et al., 2018). It is typically assessed through performance in activities of daily living (ADL; Katz et al., 1963) and instrumental activities of daily living (IADL; Lawton and Brody, 1969), with ADL referring to more basic activities (e.g., walking, eating, bathing) than IADL (e.g., shopping, preparing meal, housekeeping). Older adults who perform these activities without limitations have life expectancies 5 years longer than those with functional limitations (Keeler et al., 2010) and are more satisfied with their life (Cummings, 2002). Understanding the mechanisms underlying the associations between adverse childhood experiences and functional dependence could reveal new ways to increase life expectancy and well-being. 
Depression, a mood disorder that causes a persistent feeling of sadness and loss of interest (American Psychiatric Association, DSM-5 Task Force, 2013), may be critical in understanding the relation between adverse childhood experiences and functional dependence. Indeed, etiological models for depression consider stressful experiences, such as adverse childhood events, as depression triggers (Monroe and Simons, 1991). Recent results supported these models by showing a strong positive relationship between adverse childhood experiences and the probability of depressive disorders in late adulthood (Chapman et al., 2004; Angelini et al., 2016), thereby supporting the theoretical link between adverse childhood experiences and depression. Additionally, studies have shown an association between depression and functional dependence (Braune and Berger, 2005; Ormel et al., 1994; Shrira and Litwin, 2014; Wada et al., 2005). The mechanisms underlying this association could lie in the main symptom of depression, the "lack of interest to all or most things" (Rosenström and Jokela, 2017), which can potentially decrease the frequency an individual engages in ADL and IADL. On In the long term, this decreased frequency could impact the ability to perform these activities, especially in aging, by further increasing the age-related decline of movement control such as postural balance (Boisgontier et al., 2017) and bimanual coordination (Maes et al., 2017).

In sum, data support the effect of adverse childhood experiences on functional dependence and depression, and depression on functional dependence, thereby suggesting that depression could mediate the effect of adverse childhood experiences on functional dependence. However, to the best of our knowledge, no study has formally tested for this potential mechanism.

While understanding the mechanisms underlying the relation between adverse childhood experiences and health outcomes is important from a fundamental research perspective, this knowledge could inform clinical and political decisions aiming at improving health. To address this point, we investigated whether protective factors could attenuate the relations linking adverse childhood experiences, depression, and functional dependence. We focused on physical activity, a low-cost and safe factor shown to benefit healthy aging (Bauman et al., 2016). Physical activity reduces the risk of developing depression (Chekroud et al., 2018; Teychenne et al., 2010) and decreases functional dependence (Tak et al., 2013). Therefore, physical activity could have a protective effect on functional dependence by attenuating the association between adverse childhood experiences and depression, and between depression and functional dependence. To the best of our knowledge, no study has yet examined this possibility.

This study aimed to examine whether depression explains the effect of adverse childhood experiences on functional dependence in older age and whether physical activity can reduce this negative impact of adverse childhood experiences. We hypothesized that the effect of adverse childhood experiences on functional dependence is mediated by depression and that this mediation is moderated by physical activity.

\section{METHODS}

\subsection{Study population and design}

Our analyses used data from the Survey of Health, Ageing and Retirement in Europe (SHARE), a longitudinal (12 years: 2004-2015; 6 waves; data collection every 2 years), cross-national (14 European countries) study of adults aged 50 years or older (Börsch-Supan et al., 2013). Functional dependence in ADL and IADL, depression and physical activity were assessed at the first, second, fourth, fifth, and sixth waves. Retrospective adverse childhood experiences were assessed at the third wave. Participants included in the analysis were aged 50 to 96 years and had completed the third wave with at least one measure of ADL (or IADL), depression, and physical activity. SHARE was approved by the relevant research ethics committees in the participating countries, and all participants provided written informed consent.

\subsection{Variables}

\subsubsection{Predictor: Adverse childhood experiences}

Adverse childhood experiences are traumatic events that affect children or adolescents directly or indirectly through their living environments and are out of a child's or adolescent's control (Felitti et al., 1998; Hughes et al., 2017). We carefully screened SHARE for variables that matched with this definition of adverse childhood experiences and included 6 binary indicators reflecting specific adverse childhood experiences 
before the age of 16 years: child in care (living in a children's home or with a foster family), parental death (father, mother, or both), parental mental illness, parental drinking abuse, period of hunger, and property taken away. By combining the information of these 6 indicators, we computed a 3-level categorical variable of participants with no adverse childhood experience (i.e., participants who answered "no" to all categories filled out), 1 adverse childhood experience (i.e., participants who answered "yes" to only 1 category filled out), and with 2 or more adverse childhood experiences (i.e., participants who answered "yes" to at least 2 categories filled out). Participants with missing data for all 6 categories were excluded. When data were missing for adverse childhood experiences, the score was computed based on the available indicators to keep the maximum number of participants in the analyses (Solís et al., 2015).

\subsubsection{Outcomes: Functional dependence}

Two outcomes were used to measure functional dependence: the number of functional limitations in ADL and IADL. The measure of ADL based on the validated index of Katz et al. (1963) included 6 everyday self-care activities: dressing (including putting on shoes and socks), walking across a room, bathing or showering, eating, getting in or out of bed, and using the toilet (including getting up or down). The number of functional limitations in ADL was summed up resulting in a score ranging from 0 to 6 . Higher scores were indicative of higher functional dependence (Landös et al., In press).

The measure of IADL based on the validated index of Lawton and Brody (1969) included 7 activities: using a map to figure out how to get around in a strange place, preparing a hot meal, shopping for groceries, making telephone calls, taking medications, doing work around the house or garden, and managing money (such as paying bills and keeping track of expenses). Scores ranged from 0 to 7 and higher scores were indicative of higher functional dependence (Landös et al., In press).

\subsubsection{Mediator: Depression}

Depression was measured using the EURO-D scale (Copeland et al., 2004; Prince et al., 1999) including 12 items: depressed mood, pessimism, wishing death, guilt, sleep, interest, irritability, appetite, fatigue, concentration, enjoyment, and tearfulness. Each item was scored 0 (symptom not present) or 1 (symptom present), generating an ordinal scale ranging from 0 to 12 .

\subsubsection{Moderator: Physical activity}

Participants who engaged in activities that require vigorous, moderate, or low level of energy more than once a week were classified as physically active and the other participants were classified as inactive (Cheval et al., 2018). Specifically, two items were used to assess the usual level of physical activity over a week (Lindwall et al., 2011; de Souto Barreto et al., 2017). The first item assessed vigorous physical activity ("How often do you engage in vigorous physical activity, such as sports, heavy housework, or a job that involves physical labor?"). The second item assessed moderate physical activity ("How often do you engage in activities that require a low or moderate level of energy such as gardening, cleaning the car, or doing a walk?"). Participants answered by using a four-point scale ( $1=$ more than once a week; $2=$ once a week; 3 $=$ one to three times a month; $4=$ hardly ever, or never). Participants who answered "1" to either or both items were classified as physically active and the other participants were classified as inactive (Cheval et al., 2018).

\subsubsection{Covariates}

We considered age at baseline, wave (i.e., time), gender (male, female), country of residence (Austria, Belgium, Czech Republic, Denmark, France, Germany, Greece, Ireland, Italy, Netherlands, Poland, Spain, Sweden, Switzerland), birth cohort (1914-1918 and 1939-1945 wars, 1929-1938 Great Depression, no war and no economic crisis: before 1913, between 1919 and 1928, and after 1945), participant attrition (dropout, i.e., participants who did not respond to both waves 5 and 6; death, i.e., participants who died during the survey), childhood socioeconomic circumstances (Supplementary Material), and childhood health problems (Supplementary Material) as covariates. 


\subsection{Statistical analyses}

\subsubsection{Mediation by depression}

The mediation effect of depression on the association between adverse childhood experiences and functional dependence was analyzed using linear mixed-effect models, which account for the nested structure of the data (e.g., multiple observations within a single participant), thereby providing accurate parameter estimates with acceptable type I error rates (Boisgontier and Cheval, 2016). The random structure encompassed random intercepts for participants and random linear slopes for waves at the level of participants. These random slopes estimated each participant's linear growth trajectory over the waves of the study (i.e., 12-years). The mediation was assessed using the component approach (Yzerbyt et al., In press). To demonstrate mediation, three models needed to be tested (Baron and Kenny, 1986; Judd and Kenny, 1981; Yzerbyt et al., In press). Model 1 (Equation 1) tested the effect of adverse childhood experiences on functional dependence, adjusting for prior confounders. This model included interaction terms between age at baseline and time (linear and quadratic) to adjust for the influence of age on the rate of increasing dependence over the waves. Waves were centered on the first wave of SHARE to estimate the main effects at the baseline assessment. Quadratic time accounted for potential accelerated rates of increasing functional dependence over the waves. Model 2 (Equation 2) tested the effect of adverse childhood experiences on depression. In Model 3 (Equation 3), depression (the potential mediator) and its interaction with waves (linear and quadratic) were added to Model 1.

$$
\begin{aligned}
& \text { Functional Dependence } e_{i j} \\
& =\left(\beta_{0}+\theta_{0 j}\right)+\beta_{1} \text { Age }_{j} \times\left(\beta_{2}+\gamma_{2 j}\right) \text { Wave }_{i j} \\
& +\beta_{1} \text { Age }_{\mathrm{j}} \times \beta_{3} \text { Squared Wave }_{\mathrm{ij}}+\beta_{4} \text { Gender }_{\mathrm{j}}+\beta_{5} \text { Country }_{\mathrm{j}}
\end{aligned}
$$

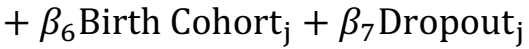

$$
\begin{aligned}
& +\beta_{8} \text { Childhood Socioeconomic Circumstances } \mathrm{j}_{\mathrm{j}} \\
& +\beta_{9} \text { Childhood Health Problems } \\
& +\beta_{10} \text { Adverse Childhood Experience }_{\mathrm{j}}+\epsilon_{i j} \\
& \text { Depression }_{i j}=\left(\beta_{0}+\theta_{0 j}\right)+\beta_{1} \text { Age }_{\mathrm{j}} \times\left(\beta_{2}+\gamma_{2 j}\right) \text { Wave }_{\mathrm{ij}} \\
& +\beta_{1} \text { Age }_{\mathrm{j}} \times \beta_{3} \text { Squared Wave }_{\mathrm{ij}}+\beta_{4} \text { Gender }_{\mathrm{j}}+\beta_{5} \text { Country }_{\mathrm{j}}
\end{aligned}
$$

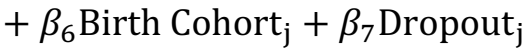

$$
\begin{aligned}
& +\beta_{8} \text { Childhood Socioeconomic } \text { Circumstances }_{\mathrm{j}} \\
& +\beta_{9} \text { Childhood Health Problems } \\
& +\beta_{10} \text { Adverse Childhood Experience }{ }_{\mathrm{j}}+\epsilon_{i j}
\end{aligned}
$$

Functional Dependence $_{i j}$

$$
\begin{aligned}
& =\left(\beta_{0}+\theta_{0 j}\right)+\beta_{1} \text { Age }_{\mathrm{j}} \times\left(\beta_{2}+\gamma_{2 j}\right)_{\text {Wave }_{i j}} \\
& +\beta_{1} \text { Age }_{\mathrm{j}} \times \beta_{3} \text { Squared Wave }_{\mathrm{ij}}+\beta_{4} \text { Gender }_{\mathrm{j}}+\beta_{5} \text { Country }_{\mathrm{j}} \\
& +\beta_{6} \text { Birth Cohort }_{\mathrm{j}}+\beta_{7} \text { Dropout }_{\mathrm{j}} \\
& +\beta_{8} \text { Childhood Socioeconomic Circumstances }_{\mathrm{j}} \\
& +\beta_{9} \text { Childhood Health Problems }_{\mathrm{j}} \\
& +\beta_{10} \text { Adverse Childhood Experiences }_{\mathrm{j}}+\beta_{11} \text { Depression }_{\mathrm{j}} \times\left(\beta_{2}\right. \\
& \left.+\gamma_{2 j}\right) \text { Wave }_{\mathrm{ij}}+\beta_{11} \text { Depression }_{\mathrm{j}} \times \beta_{3} \text { Squared Wave }_{\mathrm{ij}}+\epsilon_{i j}
\end{aligned}
$$

where Functional Dependence ${ }_{i j}$ is the $\mathrm{j}^{\text {th }}$ participant's ADL or IADL dependence score on wave $\mathrm{i}, \beta_{0}$ to $\beta_{10}$ are the fixed effect coefficients, $\theta_{0 j}$ is the random effect for the $\mathrm{j}^{\text {th }}$ participant (random intercept), $\gamma_{2 j}$ is the random effect for wave for the $\mathrm{j}^{\text {th }}$ participant (random slope), and $\epsilon_{i j}$ is the error term. 
To examine whether depression mediated the effect of adverse childhood experiences on functional dependence, four conditions must be met (Baron and Kenny,1986; Judd and Kenny, 1981; Muller et al., 2015). In Equation 1, there must be a significant effect of adverse childhood experiences on functional dependence. In Equation 2, there must be a significant effect of adverse childhood experiences on depression. In Equation 3, there must be an effect of depression on functional dependence, controlling for the effect of adverse childhood experiences. Finally, in Equation 3, the residual direct effect of adverse childhood experiences on functional dependence should be smaller than the effect of adverse childhood experiences in Equation 1. This component approach to the assessment of mediation was preferred over the index approach (Hayes, 2013), because the later has shown higher risk of false positives (Type I errors; Yzerbyt et al., In press). The percentage of decrease in the effect of adverse childhood experiences on functional dependence between the models without (1) and with (3) the mediating variable was calculated as follows: $\left(b_{\text {model1 }}-b_{\text {model } 3}\right) \times\left(b_{\text {model1 }}\right)^{-1} \times 100$. These percentages provided an estimate of the proportional influence of the potential mediating variable (depression) on the relation between adverse childhood experiences and functional dependence.

\subsubsection{Moderation by physical activity}

The moderating effect of physical activity on the direct and indirect association between adverse childhood experiences and functional dependence was analyzed using three models (Muller et al., 2015). Adding an adverse childhood experiences $\times$ physical activity interaction term in Equation 1 allowed the overall effect of adverse childhood experiences on functional dependence (Model 4) to be moderated by physical activity. Adding an adverse childhood experiences $\times$ physical activity interaction term in Equation 2 allowed the effect of adverse childhood experiences on depression (Model 5) (i.e., the first stage of the mediating processes) to be moderated by physical activity. Including interaction terms with physical activity in Equation 3 allowed the effect of depression (i.e., the second stage of the mediating processes) and the residual effect of adverse childhood experiences on functional dependence (Model 6) to be moderated by physical activity.

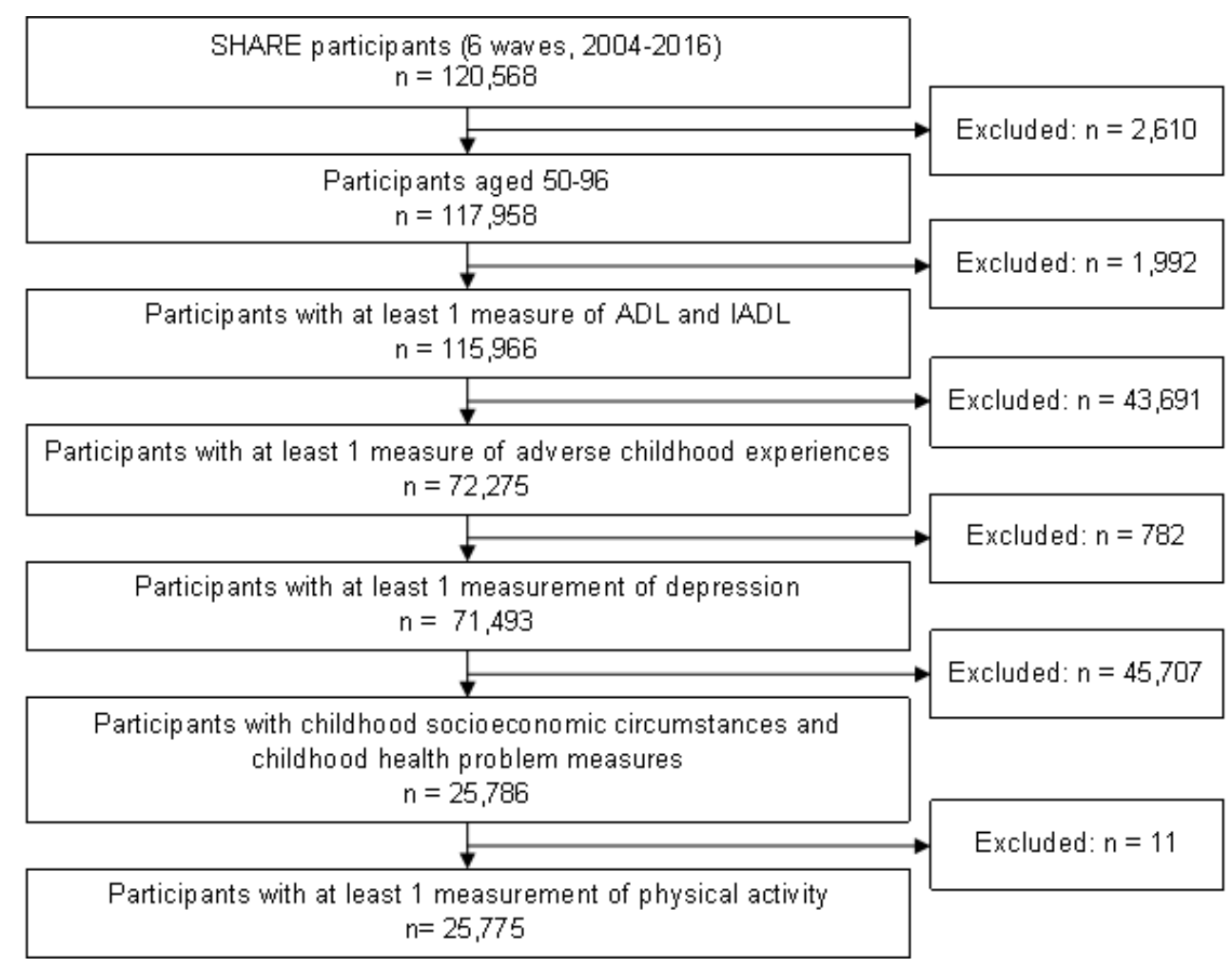

Figure 1. Flow diagram. 


\section{RESULTS}

\subsection{Descriptive results}

The dataset included a total of 25,775 participants, aged $62 \pm 9$ years (mean \pm SD), living in 14 European countries, with a depression score of $2.3 \pm 2.2,14,342$ women $(55.6 \%), 18,080$ physically active participants (70.1\%), 20,263 participants (78.6\%) without adverse childhood experience, 4,526 (17.6\%) with 1 adverse childhood experience, and $986(3.8 \%)$ with 2 or more childhood adverse experiences. The flow diagram is reported in Figure 1.

\subsection{Effects of adverse childhood experiences on functional dependence}

\subsubsection{Mediation by depression}

\subsubsection{1. $A D L$}

Model 1 (Equation 1) showed a graded association between the number of adverse childhood experiences ( 0 vs. 1,0 vs. $\geq 2)$ and the number of functional limitations in ADL ( $\mathrm{bs}=.040, .067, p \mathrm{~s}=6 \times 10^{-6}, 1 \times 10^{-4}$, 95\% CIs [.023, .057], [.033, .101], respectively; Figure 2, left panel). Model 2 (Equation 2) showed an association between the number of adverse childhood experiences ( 0 vs. 1,0 vs. $\geq 2)$ and the number of depressive symptoms in older age $\left(\mathrm{bs}=.361, .599, \mathrm{ps}<2 \times 10^{-16}, 95 \%\right.$ CIs [.307, .415], [.492, .705], respectively; Figure 2, middle panel). Model 3 (Equation 3) showed that a larger number of depressive symptoms was associated with a larger number of limitations in ADL $\left(b=.057, p<2 \times 10^{-16}, 95 \%\right.$ CIs [.082, .087]; Figure 2, right panel). Model 3 also showed that the graded association between the number of adverse childhood experiences ( 0 vs. 1,0 vs. $\geq 2$ ) and the number of functional limitations in ADL became weaker with the addition of depression ( $\mathrm{bs}=.019, .033, \mathrm{ps}=.023, .048,95 \%$ CIs $[.003, .036],\left[2 \times 10^{-4}\right.$, .067], respectively; Figure 2, left panel). From model 1 to 3 , these associations decreased by $51 \%$ ( 0 vs. 1 adverse childhood experience) and $50 \%$ ( 0 vs. $\geq 2$ adverse childhood experiences). Taken together, these results demonstrate that depression mediated the association between adverse childhood experiences and functional dependence in ADL.

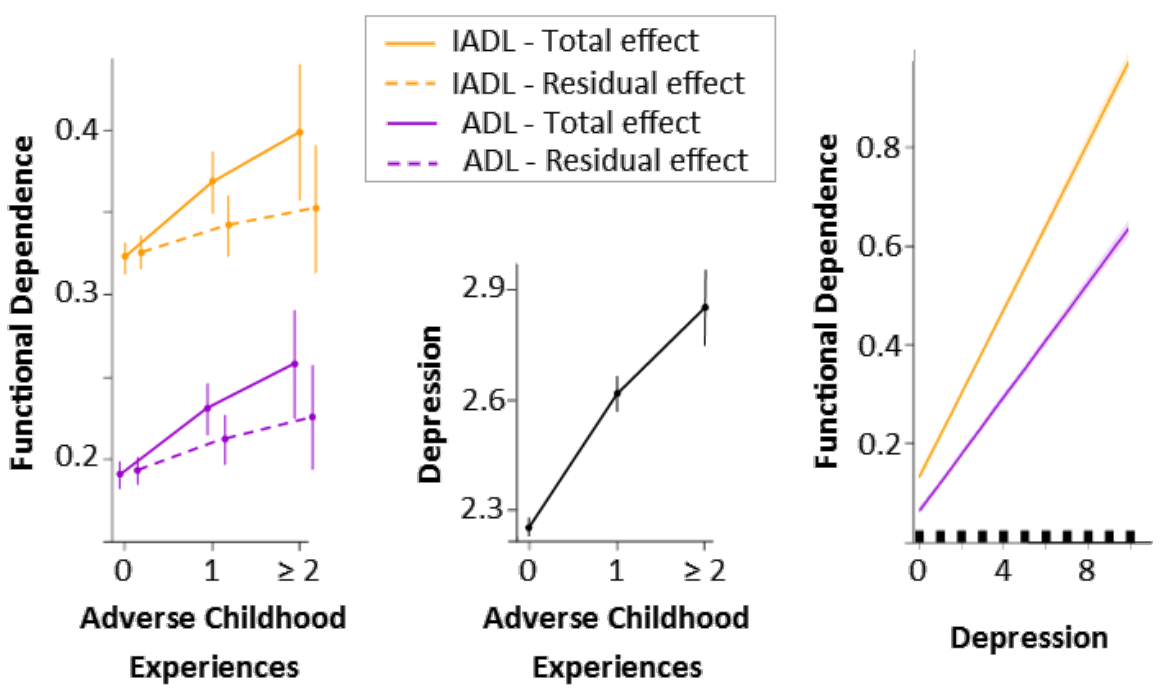

Figure 2. Main effects and $\mathbf{9 5 \%}$ confidence interval demonstrating that depression mediates the association between adverse childhood experiences and functional dependence in activities of daily living (ADL; orange) and instrumental activities of daily living (IADL; purple). Solid lines illustrate the effect of adverse childhood experiences when the mediator (depression) is not in the equation (total effect). Dashed lines illustrate the effect of adverse childhood experiences when depression is in the equation (residual effect).

\subsubsection{IADL}

Model 1 showed a graded association between the number of adverse childhood experiences $(0 \mathrm{vs} .1,0 \mathrm{vs}$. $\geq 2$ ) and the number of functional limitations in ADL ( $\mathrm{bs}=.046, .076, p \mathrm{~s}=2 \times 10^{-5}, 4 \times 10^{-4}, 95 \% \mathrm{CIs}[.025$, 
.067], [.034, .117], respectively; Figure 2, left panel). Model 2 was the same as for ADL (Figure 2, middle panel). Model 3 showed that a larger number of depressive symptoms was associated with a larger number of limitations in IADL $\left(\mathrm{b}=.085, p<2 \times 10^{-16}, 95 \%\right.$ CIs [.082, .087]; Figure 2, right panel). Model 3 also showed that the graded association between the number of adverse childhood experiences $(0 \mathrm{vs} .1,0$ vs. $\geq 2$ ) and the number of functional limitations in IADL became non-significant with the addition of depression (bs $=.016, .027, p \mathrm{~s}=.119, .184,95 \%$ CIs [-.004, .036], [-.013, .067], respectively; Figure 2, left panel). From model 1 to 3, these associations decreased by 65\% (0 vs. 1 adverse childhood experience) and $64 \%$ ( 0 vs. $\geq 2$ adverse childhood experiences). Taken together, these results demonstrate that depression mediated the association between adverse childhood experiences and functional dependence in IADL.
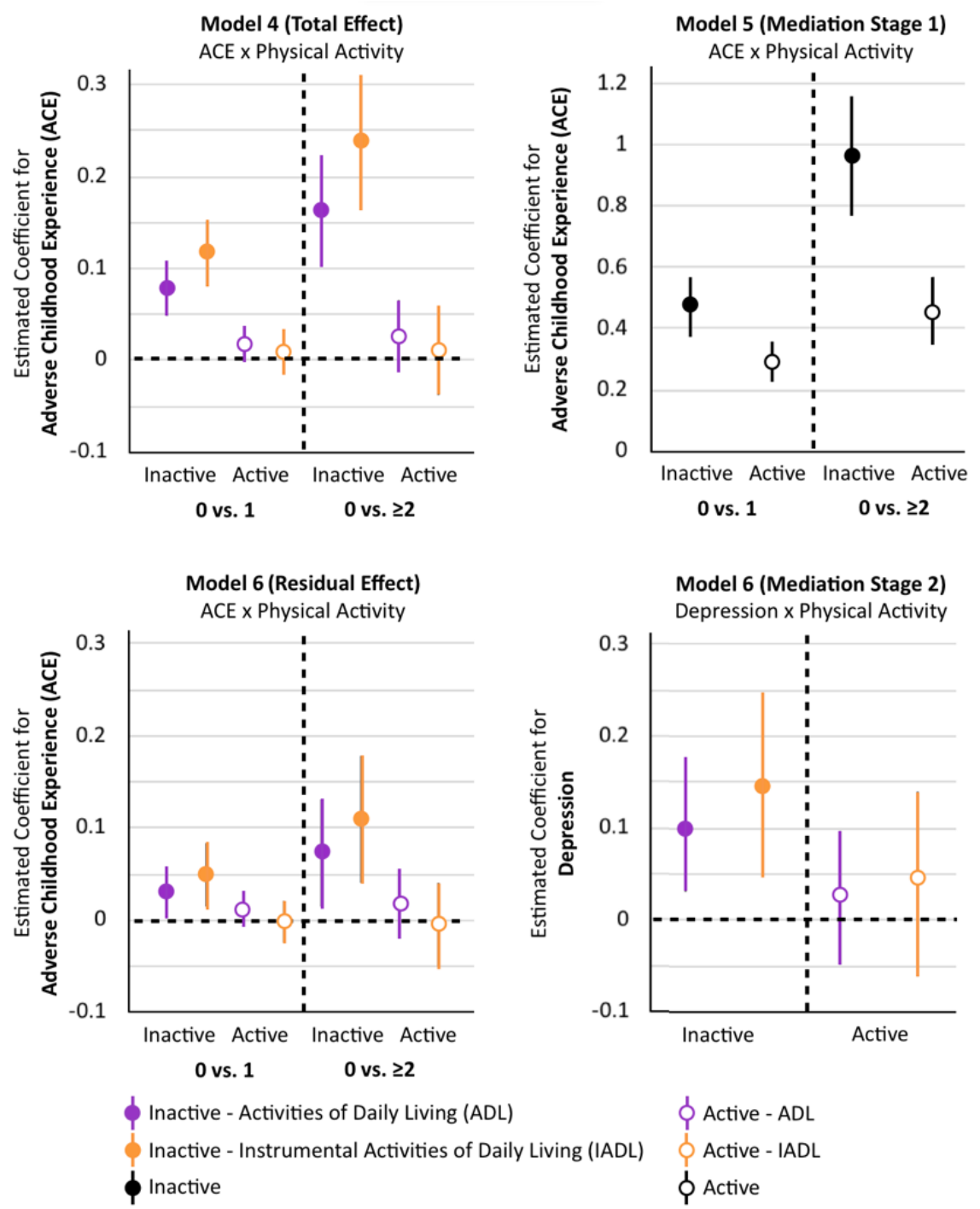

Figure 3. Conditional b coefficients and $\mathbf{9 5 \%}$ confidence interval as a function of physical activity. The upper-left panel illustrates how physical activity moderates the total effect of adverse childhood experiences ( 0 vs. 1 and 0 vs. $\geq 2$ adverse childhood experiences) on functional dependence. The upperright panel illustrates how physical activity moderates the first stage of the mediation by depression. The lower-left panel illustrates the absence of moderation by physical activity on the residual effect of adverse childhood experiences on activities of daily living (ADL) and the moderation effect on this effect on instrumental activities of daily living (IADL). The lower-right panel illustrates how physical activity moderates the second stage of the mediation. Functional dependence in ADL and IADL are in orange and purple, respectively. Physically inactive and active participants are illustrated by filled and empty circles, respectively. 


\subsubsection{Moderation by physical activity}

\subsubsection{1. $A D L$}

Model 4 showed a graded moderation of the association between adverse childhood experiences $(0$ vs. 1,0 vs. $\geq 2$ ) and functional dependence in ADL by physical activity ( $b s=-.060,-.135, p \mathrm{~s}=.001,2 \times 10^{-4}, 95 \%$ CIs [-.096, -.024], [-.207, -.063], respectively; Figure 3A, left panel). Model 5 showed a graded moderation of the association between adverse childhood experiences ( 0 vs. 1,0 vs. $\geq 2$ ) and the number of depressive syndromes by physical activity (bs $=-.179,-.515, p \mathrm{~s}=.002,9 \times 10^{-6}, 95 \%$ CIs [-.292, -.065], [-.742, -.288], respectively; Figure 3A, central panel). Model 6 showed a moderation of the association between depression and functional dependence in ADL by physical activity $\left(\mathrm{b}=-.073, p<2 \times 10^{-16}, 95 \%\right.$ CIs [-.077, -.069]; Figure $3 \mathrm{~A}$, right panel). Model 6 also showed that the graded moderation of the association between adverse childhood experiences ( 0 vs. 1,0 vs. $\geq 2)$ and functional dependence in ADL by physical activity became non-significant ( $\mathrm{bs}=-.018,-.055, \mathrm{ps}=.306, .116,95 \%$ CIs [-.053, .016], [-.124, .014], respectively). From model 4 to 6 , these moderations decreased by $70 \%$ ( 0 vs. 1 adverse childhood experience) and $59 \%$ ( 0 vs. $\geq 2$ adverse childhood experiences). Taken together these results demonstrate a mediated moderation (Muller et al., 2015; Figure 4). Figure 3 revealed that the effects of adverse childhood experiences (left panel) and depression (right panel) on functional dependence in ADL (purple in the figure) were not significant in physically active participants $(p$ s $>.081)$ as the $95 \%$ confidence interval crossed the zero line.

\subsubsection{2. $I A D L$}

Model 4 showed a graded moderation of the association between adverse childhood experiences ( 0 vs. 1,0 vs. $\geq 2$ ) and functional dependence in IADL by physical activity (bs $=-.109,-.227, p \mathrm{~s}=1 \times 10^{-6}, 4 \times 10^{-7}$, 95\% CIs [-.153, -.065], [-.315, -.140], respectively; Figure 3B, left panel). Model 5 was the same as for ADL. Model 6 showed a moderation of the association between depression and functional dependence in IADL by physical activity ( $\mathrm{b}=-.100, p<2 \times 10^{-16}, 95 \%$ CIs [-.106, -.095]; Figure 3B, right panel). Model 6 also showed that the graded moderation of the association between adverse childhood experiences $(0 \mathrm{vs.}$ 1,0 vs. $\geq 2$ ) and functional dependence in IADL by physical activity became weaker (bs = -.052, -.114, $p$ s $=.014, .007,95 \%$ CIs [-.197, -.010], [-.093, -.032], respectively). From model 4 to 6, these moderations decreased by $53 \%$ ( 0 vs. 1 adverse childhood experience) and $50 \%$ ( 0 vs. $\geq 2$ adverse childhood experiences). Taken together these results demonstrate a mediated moderation (Muller et al., 2015) (Figure 4). Figure 3 revealed that the effects of adverse childhood experiences (left panel) and depression (right panel) on functional dependence in IADL (orange in the figure) were not significant in physically active participants $(p s>.528)$.

\section{DISCUSSION}

\subsection{Main findings}

This study uncovered three main findings. For the first time, we show that depression mediates the effect of adverse childhood experiences on functional dependence in older age. Second, results showed that physical activity reduced the effect of adverse childhood experiences on depression and on the residual effect on IADL. Third, results revealed that adverse childhood experiences were not (directly or indirectly) associated with functional dependence (ADL or IADL) in physically active individuals.

\subsection{Comparison with previous studies}

Our results showing that adverse childhood experiences are associated with functional dependence is consistent with previous literature (Laditka and Laditka, 2018; Amemiya et al., 2018). The effect of adverse childhood experiences is consistent with etiological models for depression considering stressful experiences as depression triggers (Monroe and Simons, 1991) and with previous results showing an association between adverse childhood experiences and the probability of depressive disorders (Chapman et al., 2004; Angelini et al., 2016). The effect of depression on functional dependence supports previous studies showing an association between depression and functional dependence (Braune and Berger, 2005; Ormel et al., 1994; Shrira and Litwin, 2014; Wada et al., 2005). The mechanisms underlying this association could lie in the main symptom of depression, the "lack of interest to all or most things" (Rosenström and Jokela, 2017), 
which can potentially decrease the frequency an individual engages in ADL and IADL. In the long term, this decreased frequency could impact the ability to perform these activities, especially in aging, by further increasing the age-related decline of movement control such as postural balance (Boisgontier et al., 2017) and bimanual coordination (Maes et al., 2017). The protective effect of physical activity is in line with multiple studies, especially those showing that physical activity reduces the risk of developing depression (Chekroud et al., 2018) and decreases functional dependence (Tak et al., 2013).

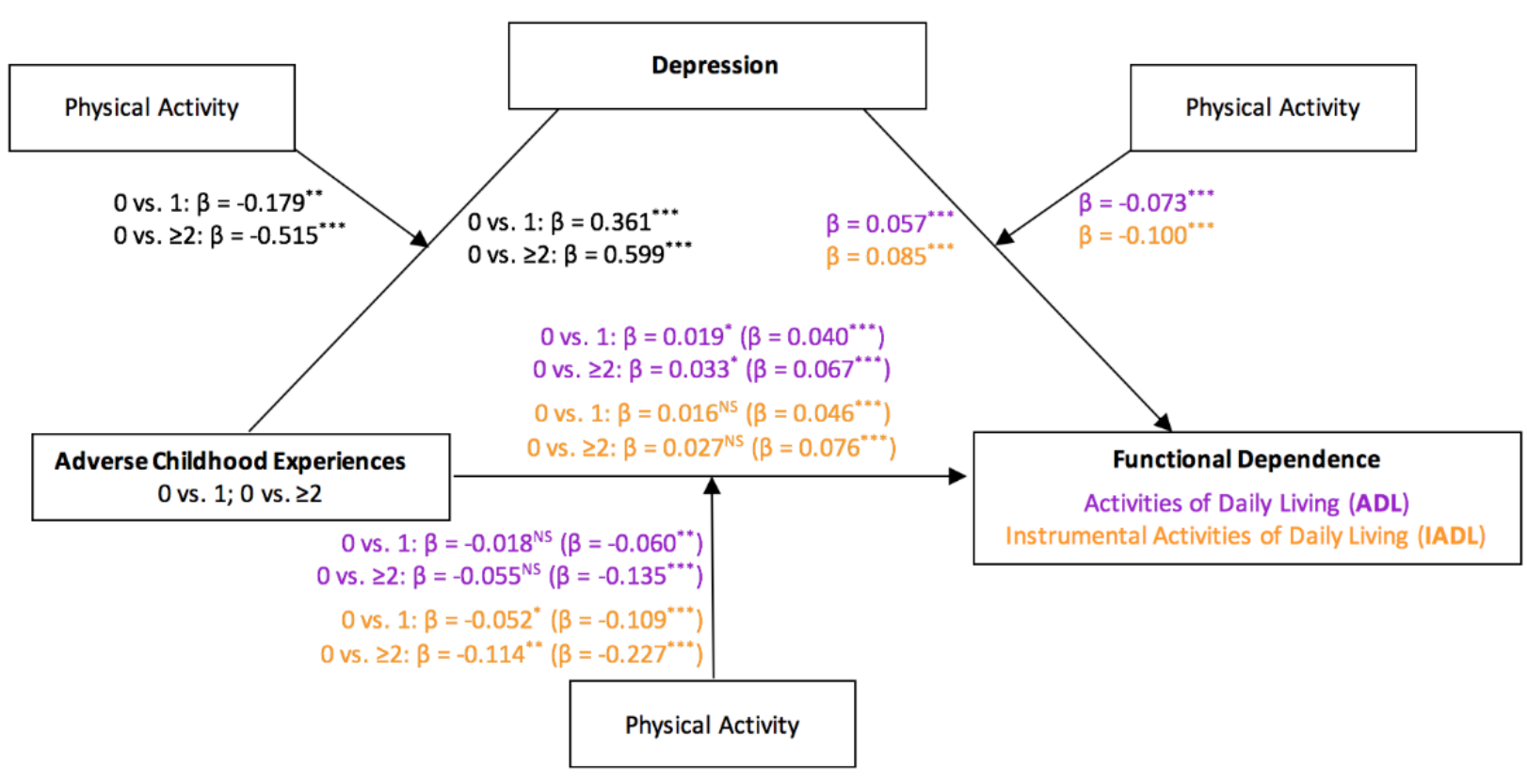

Figure 4. Mediated moderation of the effect of adverse childhood experiences on functional dependence. $\beta \mathrm{s}$ in brackets are the coefficients when the mediator is not in the equation (total effect). $\beta \mathrm{s}$ not in brackets are the coefficients when the mediator (depression) and covariates are in the equation (residual effect). Results related to functional dependence in activities of daily living (ADL) and instrumental activities of daily living (IADL) are in purple and orange, respectively.

\subsection{Strengths and limitations}

This study has many strengths. The sample is large and includes non-institutionalized older adults from multiple European countries. Moreover, we used a validated measure of depression (EURO-D), two different indicators of functional dependence that consistently showed that same pattern of results based on sophisticated statistical analyses suited to examine, for the first time, a potential mechanism of moderated mediation between adverse childhood experiences, depression, functional dependence, and physical activity. However, three potential limitations should be noted. First, there is the potential for recall bias as adverse childhood experiences were based on self-reports. Yet, the validity of these self-reports has been demonstrated (Hardt and Rutter, 2004). Second, although two widely validated measures of functional dependence were used and revealed consistent results, these measures were self-reported. Future studies should investigate whether our results apply to objective indicators of functional dependence (e.g., walking speed, chair stand, balance). There is the potential for misclassification bias regarding physical activity. However, the likelihood of this bias is small because we used a very conservative definition of physical activity.

\subsection{Conclusion}

Physical activity cancels the negative impact of adverse childhood experiences on functional dependence. In inactive individuals, the effect of adverse childhood experiences on functional dependence (ADL and IADL) is mediated by depression. 


\section{REFERENCES}

Amemiya A, Fujiwara T, Murayama H, Tani Y, Kondo K. Adverse childhood experiences and higher-level functional limitations among older Japanese people: results from the JAGES study. J Gerontol A Biol Sci Med Sci. 2018;73:261-266. https://doi.org/10.1093/gerona/glx097

American Psychiatric Association, DSM-5 Task Force. Diagnostic and statistical manual of mental disorders: DSM-5 ${ }^{\mathrm{TM}}$. 5th ed. Arlington, VA, US: American Psychiatric Publishing, Inc.; 2013. http://dx.doi.org/10.1176/appi.books.97808904255963

Angelini V, Klijs B, Smidt N, Mierau JO. Associations between childhood parental mental health difficulties and depressive symptoms in late adulthood: the influence of life-course socioeconomic, health and lifestyle factors. PLoS One. 2016;11:e0167703. https://doi.org/10.1371/journal.pone.0167703

Baron RM, Kenny DA. The moderator-mediator variable distinction in social psychological research: conceptual, strategic, and statistical considerations. J Pers Soc Psychol. 1986;51:1173-1182. http://dx.doi.org/10.1037/0022-3514.51.6.1173

Bauman A, Merom D, Bull FC, Buchner DM, Fiatarone Singh MA. Updating the evidence for physical activity: summative reviews of the epidemiological evidence, prevalence, and interventions to promote "active aging". Gerontologist. 2016;56:S268-S280. https://doi.org/10.1093/geront/gnw031

Boisgontier MP, Beets IAM, Duysens J, Nieuwboer A, Krampe RT, Swinnen SP. Age-related differences in attentional cost associated with postural dual tasks: increased recruitment of generic cognitive resources in older adults. Neurosci Biobehav Rev. 2013;37:1824-1837. https://doi.org/10.1016/j.neubiorev.2013.07.014

Boisgontier MP, Cheval B. The anova to mixed model transition. Neurosci Biobehav Rev. 2016 Sep;68:1004-1005. https://doi.org/10.1016/j.neubiorev.2016.05.034

Börsch-Supan A, Brandt M, Hunkler C, Kneip T, Korbmacher J, Malter F, et al. Data resource profile: the Survey of Health, Ageing and Retirement in Europe (SHARE). Int J Epidemiol. 2013;42:992-1001. https://doi.org/10.1093/ije/dyt088

Braune BT, Berger K. The influence of depressive mood on activities of daily living and health care utilization in the elderly - the MEMO study on the KORA platform Augsburg. Gesundheitswesen. 2005;67:S176-S179. https://doi.org/10.1055/s-2005-858236

Chapman DP, Whitfield CL, Felitti VJ, Dube SR, Edwards VJ, Anda RF. Adverse childhood experiences and the risk of depressive disorders in adulthood. $J$ Affect Disord. 2004;82:217-225. http://dx.doi.org/10.1016/j.jad.2003.12.013

Chekroud SR, Gueorguieva R, Zheutlin AB, Paulus M, Krumholz HM, Krystal JH, et al. Association between physical exercise and mental health in 1.2 million individuals in the USA between 2011 and 2015: a cross-sectional study. Lancet Psychiatry. 2018. doi: 10.1016/S2215-0366(18)30227-X

Cheval B, Sieber S, Guessous I, Orsholits D, Courvoisier DS, Kliegel M, et al. Effect of early- and adultlife socioeconomic circumstances on physical inactivity. Med Sci Sports Exerc. 2018;50:476-485. https://doi.org/10.1249/mss.0000000000001472

Copeland JRM, Beekman ATF, Braam AW, Dewey ME, Delespaul P, Fuhrer R, et al. Depression among older people in Europe: the EURODEP studies. World Psychiatry. 2004;3:45-49.

Cummings SM. Predictors of psychological well-being among assisted-living residents. Health Soc Work. 2002;27:293-302. https://doi.org/10.1093/hsw/27.4.293

de Souto Barreto P, Cesari M, Andrieu S, Vellas B, Rolland Y. Physical activity and incident chronic diseases: a longitudinal observational study in 16 European countries. Am J Prev Med. 2017;52:373378. https://doi.org/10.1016/j.amepre.2016.08.028

Felitti VJ, Anda RF, Nordenberg D, Williamson DF, Spitz AM, Edwards V, et al. Relationship of childhood abuse and household dysfunction to many of the leading causes of death in adults: the Adverse Childhood Experiences (ACE) Study. Am J Prev Med. 1998;14:245-258. https://doi.org/10.1016/S0749-3797(98)00017-8 
Hardt J, Rutter M. Validity of adult retrospective reports of adverse childhood experiences: review of the evidence. J Child Psychol Psychiatry 2004;45:260-273. https://doi.org/10.1111/j.14697610.2004.00218.x

Hayes AF. Introduction to mediation, moderation, and conditional process analysis: a regression-based approach. 2013; New York: Guilford Press.

Hughes K, Bellis MA, Hardcastle KA, Sethi D, Butchart A, Mikton C, et al. The effect of multiple adverse childhood experiences on health: a systematic review and meta-analysis. Lancet Public Health. 2017;2:e356-e366. https://doi.org/10.1016/S2468-2667(17)30118-4

Judd CM, Kenny DA. Process analysis: Estimating mediation in treatment evaluation. Eval Rev. 1981:5;602-619. https://doi.org/10.1186/s12879-014-0718-6

Katz S, Ford AB, Moskowitz RW, Jackson BA, Jaffe MW. Studies of illness in the aged. The index of ADL: a standardized measure of biological and psychosocial function. JAMA. 1963;185:914-919. https://doi.org/10.1001/jama.1963.03060120024016

Keeler E, Guralnik JM, Tian H, Wallace RB, Reuben DB. The impact of functional status on life expectancy in older persons. J Gerontol A Biol Sci Med Sci. 2010;65:727-733. https://doi.org/10.1093/gerona/glq029

Laditka JN, Laditka SB. Adverse childhood circumstances and functional status throughout adult life. J Aging Health. 2017:898264317715976. https://doi.org/10.1177/0898264317715976

Landös A, von Arx M, Cheval B, Sieber S, Kliegel M, Gabriel R, et al. Childhood socioeconomic circumstances and disability trajectories in older men and women: a European cohort study. Eur J Public Health. In press. https://doi.org/10.1093/eurpub/cky166

Lawton MP, Brody EM. Assessment of older people: self-maintaining and instrumental activities of daily living. Gerontologist. 1969;9:179-186. https://doi.org/10.1093/geront/9.3_part_1.179

Lindwall M, Larsman P, Hagger MS. The reciprocal relationship between physical activity and depression in older European adults: a prospective cross-lagged panel design using SHARE data. Health Psychol. 2011;30:453-462. https://doi.org/10.1037/a0023268

Maes C, Gooijers J, Orban de Xivry JJ, Swinnen SP, Boisgontier MP. Two hands, one brain, and aging. Neurosci Biobehav Rev. 2017;75:234-256. https://doi.org/10.1016/j.neubiorev.2017.01.052

Monroe SM, Simons AD. Diathesis-stress theories in the context of life stress research: implications for the depressive disorders. Psychol Bull. 1991;110:406-425. http://dx.doi.org/10.1037/00332909.110.3.406

Muller D, Judd CM, Yzerbyt VY. When moderation is mediated and mediation is moderated. J Pers Soc Psychol. 2005;89:852-863.

Ormel J, VonKorff M, Ustun TB, Pini S, Korten A, Oldehinkel T. Common mental disorders and disability across cultures. Results from the WHO Collaborative Study on Psychological Problems in General Health Care. JAMA. 1994;272:1741-1748. https://doi.org/10.1001/jama.1994.03520220035028

Prince MJ, Reischies F, Beekman ATF, Fuhrer R, Jonker C, Kivela SL, et al. Development of the EUROD scale - a European, Union initiative to compare symptoms of depression in 14 European centres. $\mathrm{Br}$ J Psychiatry. 1999;174:330-338. https://doi.org/10.1192/bjp.174.4.330

Rosenström T, Jokela M. Reconsidering the definition of major depression based on collaborative psychiatric epidemiology surveys. J Affect Disord. 2017;207:38-46. http://dx.doi.org/10.1016/j.jad.2016.09.014

Shrira A, Litwin H. The effect of lifetime cumulative adversity and depressive symptoms on functional status. J Gerontol B Psychol Sci Soc Sci. 2014;69 :953-965. doi: 10.1093/geronb/gbu05614.

Solís CB, Kelly-Irving M, Fantin R, Darnaudéry M, Torrisani J, Lang T, et al. Adverse childhood experiences and physiological wear-and-tear in midlife: findings from the 1958 British birth cohort. Proc Natl Acad Sci U S A. 2015;112:E738-E746. https://doi.org/10.1073/pnas. 1417325112

Tak E, Kuiper R, Chorus A, Hopman-Rock M. Prevention of onset and progression of basic ADL disability by physical activity in community dwelling older adults: a meta-analysis. Ageing Res Rev. 2013;12:329338. https://doi.org/10.1016/j.arr.2012.10.001 
Teychenne M, Ball K, Salmon J. Sedentary behavior and depression among adults: a review. Int J Behav Med. 2010;17:246-254. doi: 10.1007/s12529-010-9075-Z

Wada T, Ishine M, Sakagami T, Kita T, Okumiya K, Mizuno K, et al. Depression, activities of daily living, and quality of life of community-dwelling elderly in three Asian countries: Indonesia, Vietnam, and Japan. Arch Gerontol Geriatr. 2005;41:271-280. https://doi.org/10.1016/j.archger.2005.03.003

Yzerbyt VY, Muller D, Batailler C, Judd CM. New recommendations for testing indirect effects in mediational models: the need to report and test component paths. J Pers Soc Psychol. In press.

\section{AUTHOR CONTRIBUTIONS}

MPB conceived the study. SC, SS managed the data. MPB, BC analyzed the data. MPB, BC drafted the manuscript. DO prepared the code to make it suitable for open repositories. All authors contributed to the improvement of the manuscript.

\section{PREPRINT AGREEMENT}

All authors are aware of and have agreed to submission of this manuscript as a preprint.

\section{DATA AND CODE SHARING}

The SHARE dataset is freely available at http://www.share-project.org/data-access.html. The code is available on GitHub (https://github.com/matthieuboisgontier/SHARE ACE FunctionalDependence) and will soon be published in Zenodo and get a DOI.

\section{FUNDING}

This work was supported by the Swiss National Centre of Competence in Research "LIVES - Overcoming vulnerability: Life course perspectives", which is financed by the Swiss National Science Foundation (SNSF; 51NF40-160590).

\section{COMPETING INTERESTS}

All authors declare no financial relationships with any organizations that might have an interest in the submitted work in the previous three years; no other relationships or activities that could appear to have influenced the submitted work.

\section{ETHICAL APPROVAL}

This study was part of the SHARE study, approved by the relevant research ethics committees in the participating countries, and all participants provided written informed consent.

\section{TRANSPARENCY}

The lead author affirms that the manuscript is an honest, accurate, and transparent account of the study being reported, that no important aspects of the study have been omitted, and that any discrepancies from the study as planned have been explained.

\section{ACKNOWLEDGEMENTS}

This article uses data from SHARE Waves 1, 2, 3 (SHARELIFE), 4, 5, and 6 (DOIs: 10.6103/SHARE.w1.600, 10.6103/SHARE.w2.600, 10.6103/SHARE.w3.600, 10.6103/SHARE.w4.600, 10.6103/SHARE.w5.600, 10.6103/SHARE.w6.600).

The SHARE data collection was primarily funded by the European Commission through FP5 (QLK6-CT2001-00360), FP6 (SHARE-I3: RII-CT-2006-062193, COMPARE: CIT5-CT-2005-028857, SHARELIFE: CIT4-CT-2006-028812), and FP7 (SHARE-PREP: no.211909, SHARE-LEAP: no.227822, SHARE M4: no.261982). Additional funding from the German Ministry of Education and Research, the Max Planck Society for the Advancement of Science, the U.S. National Institute on Aging (U01_AG09740-13S2, P01_AG005842, P01_AG08291, P30_AG12815, R21_AG025169, Y1-AG-4553-01, IAG_BSR06-11, 
OGHA_04-064, HHSN271201300071C), and from various national funding sources is gratefully acknowledged (see www.share-project.org).

\section{SUPPLEMENTAL MATERIAL}

\section{Childhood socioeconomic circumstances}

Childhood socioeconomic circumstances were determined according to the index of childhood circumstances (Wahrendorf and Blane, 2015) combining 4 binary indicators reflecting specific childhood socioeconomic circumstances of participants at the age of 10: occupational position of the main breadwinner, number of books at home, housing overcrowding, and housing quality. The occupational position of the main breadwinner was built based on a reclassification of the 10 main occupational groups of the International Standard Classification of Occupations (ISCO) according to their skill levels (Wahrendorf et al., 2013). The first and second levels were grouped into the "disadvantaged" occupational position and the third and fourth levels were grouped into the "advantaged" occupational position. A binary item was built for the number of books at home, with the category " $0-10$ books" an indicator of social disadvantage. Overcrowding (i.e., more than 1 person per room lived in the housing) was constructed based on information related to the number of people living in the housing and number of rooms in the house (excluding kitchen, bathrooms, and hallways). Quality of the housing was assessed by the presence of a fixed bath, cold running water supply, hot running water supply, inside toilet, and central heating. If none of these were present, the housing was coded as "disadvantaged". By combining the information for these 4 indicators, we computed a 5-level categorical variable of "most advantaged", "advantaged", "middle", "disadvantaged", and "most disadvantaged" childhood socioeconomic circumstances.

\section{Childhood health problems}

Childhood health problems were long hospitalisation (i.e., hospitalization for one month or more before the age of 15), multiple hospitalizations (i.e., more than three times within a 12-month period before the age of 15), childhood illness (e.g., polio, asthma, meningitis, encephalitis), and childhood serious health conditions (severe headaches, psychiatric problem, fractures, heart trouble, cancers, and physical injury that has led to permanent handicap, disability or limitation in daily life). By combining the information for these five indicators, we computed a two-level categorical variable of participants with no childhood health problems (i.e., participants who answered "no" to all categories filled out) and participants with one or more childhood health problems (i.e., participants who answered "yes" to at least one category filled out).

\section{Supplementary references}

Wahrendorf M, Blane D. Does labour market disadvantage help to explain why childhood circumstances are related to quality of life at older ages? Results from SHARE. Aging Ment Health. 2015;19:584-594. https://doi.org/10.1080/13607863.2014.938604

Wahrendorf M, Blane D, Bartley M, Dragano N, Siegrist J. Working conditions in mid-life and mental health in older ages. Adv Life Course Res. 2013;18:16-25. https://doi.org/10.1016/j.alcr.2012.10.004 